\title{
Lapatinib monotherapy in patients with relapsed, advanced, or metastatic breast cancer: efficacy, safety, and biomarker results from Japanese patients phase II studies
}

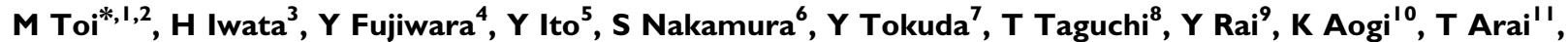 J Watanabe ${ }^{12}$, T Wakamatsu $^{13}, \mathrm{~K}_{\text {Katsura }}{ }^{13}, \mathrm{CE}_{\text {Ellis }}{ }^{14}, \mathrm{RC} \mathrm{Gagnon}^{14}, \mathrm{KE} \mathrm{Allen}^{14}$, Y Sasaki $^{15}$ and S Takashima $^{10}$}

'Department of Surgery, Graduate School of Medicine, Kyoto University, 54 Kawaracho, Shogoin, Sakyo-ku, Kyoto 606-8507, Japan; ${ }^{2}$ Department of Surgery, Tokyo Metropolitan Komagome Hospital, 3- I 8-22 Honkomagome, Bunkyo-ku, Tokyo II 3-8677, Japan; ${ }^{3}$ Department of Breast Surgery, Aichi Cancer Center Hospital, I - I Kanokoden, Chikusa-ku, Nagoya, Aichi 464-868I, Japan; ${ }^{4}$ Breast Oncology Internal Medicine, National Cancer Center Hospital, Tsukiji 5-I-1, Chuo-ku, Tokyo 104-0045, Japan; ${ }^{5}$ Department of Surgery, The Cancer Institute Hospital of JFCR, Ariake 3-10-6, Koto-ku, Tokyo 135-8550, Japan; 'Department of Breast Surgery, St Luke's International Hospital, Akashi-cho 9-1, Chuo-ku, Tokyo I04-8560, Japan; ${ }^{7}$ Breast Endocrine Surgery, Tokai University Hospital, 143 Shimokasuya, Isehara, Kanagawa 259-1 193, Japan; ${ }^{8}$ Breast Endocrine Surgery, Osaka

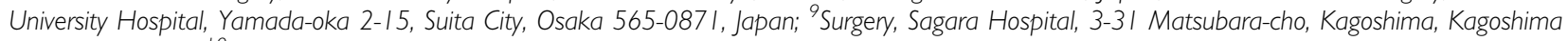
892-0833, Japan; ${ }^{10}$ Department of Surgery, Shikoku Cancer Center, 160 Kou, Minamiumemoto-cho, Matsuyama, Ehime 79I-0288, Japan;

1 'Medical Oncology, Tochigi Cancer Center, 4-9-1 3 Yonan, Utsunomiya, Tochigi 320-0834, Japan; '2 Female Internal Medicine, Shizuoka Cancer Center, 1007 Shimonagakubo, Nagaizumi, Shizuoka 4I I-8777, Japan; '3Clinical Research, GlaxoSmithKline KK, 4-6- I5, Sendagaya, Shibuya-ku I 5 I-8566, Japan; ${ }^{14}$ Clinical Oncology, GlaxoSmithKline, 1250 South Collegeville Road, Collegeville, PA 19426, USA; ${ }^{15}$ Medical oncology, Saitama Medical University, 1397-I Yamane, Hidaka, Saitama 350-I298, Japan

BACKGROUND: HER2-positive metastatic breast cancer (MBC) relapsing after trastuzumab-based therapy may require continued HER2 receptor inhibition to control the disease and preserve the patients' quality-of-life. Efficacy and safety of lapatinib monotherapy was evaluated in Japanese breast cancer patients after trastuzumab-based therapies.

METHODS: In studies, EGFI00642 and EGFI049II evaluated the efficacy and safety of oral lapatinib given I500 mg once daily in patients with advanced or MBC. All patients progressed on anthracyclines and taxanes; HER2-positive patients had also progressed on trastuzumab.

RESULTS: For HER2-positive tumours $(n=100)$, objective response rate was $19.0 \%$ (95\% confidence interval $(\mathrm{Cl})$ : $11.8-28.1)$ and clinical benefit rate (CBR) was 25.0\% (95\% Cl: 16.9-34.7). One out of 22 HER2-negative tumour was documented as complete response $(n=22)$. The median time-to-progression (TTP) in the HER2-positive and HER2-negative groups was 13.0 and 8.0 weeks $(P=0.007)$; median overall survival was 58.3 and 40.0 weeks, respectively. The most frequent adverse event was diarrhoea. TTP and CBR were significantly associated with HER2 expression. Patients with tumours harbouring an HI047R PIK3CA mutation or low expression of PTEN derived clinical benefit from lapatinib.

CONCLUSION: Lapatinib monotherapy had shown anti-tumour activity in Japanese patients with HER2-positive MBC that relapsed after trastuzumab-based therapy, including those with brain metastases. Patients benefiting from lapatinib may have biomarker profiles differing from that reported for trastuzumab.

British Journal of Cancer (2009) I I , 1676- 1682. doi:I0.1038/sj.bjc.6605343 www.bjcancer.com

Published online 20 October 2009

(C) 2009 Cancer Research UK

Keywords: lapatinib; monotherapy; HER2; metastatic breast cancer; biomarker; trastuzumab

Approximately, $30 \%$ of invasive breast cancers (BC) overexpress HER2, an adverse prognostic factor associated with aggressive histopathological parameters and worse clinical outcome (Slamon et al, 1987).

Despite the clinical breakthrough trastuzumab brought to the treatment of HER2-overexpressing BC, the majority of metastatic $\mathrm{BC}$ (MBC) does not respond to trastuzumab monotherapy, and controversy remains over the optimal therapeutic strategy after

*Correspondence: Dr M Toi; E-mail: toi@kuhp.kyoto-u.ac.jp Received 30 June 2009; revised 2 September 2009; accepted 4 September 2009; published online 20 October 2009 disease progression (Cobleigh et al, 1999; Vogel et al, 2002; Montemurro et al, 2006). Intrinsic and acquired trastuzumab resistance remains a concern (Nahta and Esteva, 2006; Xia et al, 2007). Additional concerns include increased incidence of brain metastasis and risk of both short- and long-term cardiac toxicity associated with trastuzumab therapy (Guarneri et al, 2006; Suter et al, 2007).

Lapatinib, an orally active, small molecule, reversible inhibitor of EGFR and HER2, is being evaluated for treatment of cancers with EGFR expression and HER2 overexpression, including cancers that are refractory to standard therapy. The simultaneous blockade of both receptors by lapatinib may be more effective than single receptor inhibition because of the propensity for HER family 
hetero-dimerisation and resultant signalling diversity (Mukherjee et al, 2007). Lapatinib, as monotherapy or in combination with chemotherapy, has promising anti-tumour activity in HER2 positive locally advanced or MBC as evidenced by efficacy outcome reported earlier. Japan and many other countries showed that lapatinib has efficacy in patients having HER2-positive BC with brain metastases (Xia et al, 2002). From 44 clinical trials, safety analysis indicated favourable cardiac toxicity profile of lapatinib with a low frequency of asymptomatic events that are reversible (Perez et al, 2008).

In Japan, two Phase II studies were conducted to evaluate the efficacy and safety of lapatinib monotherapy in advanced or MBC that had progressed on prior anti-tumour therapies, including trastuzumab.

\section{MATERIALS AND METHODS}

\section{Study design}

EGF100642 and EGF104911 were multicentre, single-arm, openlabel studies involving 23 centres in Japan. Studies were designed in accordance with the Japanese Ministry of Health, Labor and Welfare Ordinance on Good Clinical Practice, to evaluate the efficacy and safety of lapatinib in patients with advanced or MBC. Studies were approved by the institutional review board of each participating institution. All patients provided written informed consent.

The primary endpoint was objective response rate (ORR: confirmed complete response (CR) and partial response (PR)) according to response evaluation criteria in solid tumours (RECIST) and determined by an independent review committee. Secondary efficacy endpoints included clinical benefit rate (CBR: $\mathrm{CR}, \mathrm{PR}$ and stable disease (SD) for $\geqslant 24$ weeks); time-to-progression (TTP); and overall survival (OS). In EGF100642, patients were grouped, according to intra-tumoural HER2 expression, into cohort A or B (HER2 positive and HER2 negative, respectively). EGF104911 included patients with HER2-overexpressing tumours only. The planned accrual for EGF100642 and EGF104911 was 100 patients (40 in cohort A; 60 in cohort B) and 52 patients, respectively. Integrative analyses were conducted on efficacy, safety and biomarker data from the two studies.

\section{Patient eligibility}

Eligible patients were female aged 20-74 years with confirmed advanced (stage IIIb or IIIc with T4 lesion) or MBC that had progressed on prior anthracycline- and taxane-containing regimens. All patients with HER2 overexpression had received $\geqslant 6$ weeks of prior trastuzumab. Eligible patients had measurable lesions as defined by RECIST; an Eastern Cooperative Oncology Group (ECOG) performance status of $0-2$; left ventricular ejection fraction (LVEF) within the institutional normal range (or $\geqslant 50 \%$ ); adequate renal, hepatic and haematologic functions.

\section{Treatment schedule}

Lapatinib $1500 \mathrm{mg}$ was taken orally once daily in fasted conditions. Patients were treated until disease progression, unacceptable toxicity or consent withdrawal.

\section{Efficacy and safety assessments}

Safety and efficacy assessments (including echocardiogram for LVEF) were performed at 4- and 8-week intervals, respectively, at treatment completion and 28 days after the last dose. All patients were followed for survival every 12 weeks until death. Adverse events (AEs) were assessed according to the National Cancer
Institute Common Terminology Criteria for AEs version 3.0 (NCI CTCAE v3.0).

\section{Immunohistochemistry and fluorescent in situ hybridisation}

Formalin-fixed, paraffin-embedded (FFPE) archived tumour tissue blocks (or sections) were required for enrolment. Intra-tumoural protein expression levels were determined by immunohistochemistry (IHC) and reported as an $\mathrm{H}$-score (McCarty et al, 1986) excluding EGFR and HER2, which were reported by the assay defined ordinal score (Dako, Carpinteria, CA, USA). The following antibodies were used in the semi-quantitative IHC assays performed at Quest Diagnostics (Van Nuys, CA, USA): HER2 (HercepTest, Dako); EGFR (PharmDx, Dako) and at Pathway Diagnostics (Malibu, CA, USA): ErbB3 (NeoMarkers RB-9211, Thermo Fisher, Fremont, CA, USA); ErbB4 (\#18-7329, Zymed, South San Francisco, CA, USA); IGF1R (NeoMarkers MS-641, Thermo Fisher); BCL2 (Dako). PTEN (Cell Signaling Technology, Danvers, MA, USA, \#9559) analysis was performed at European Institute of Oncology (Milan, Italy). Deoxyribonucleic acid (DNA) fragmentation (TUNEL) was measured at Pathway Diagnostics using the ApopTag in situ assay (Chemicon International, Temecula, CA, USA). Oestrogen (ER) and progesterone $(\mathrm{PgR})$ receptor results were from local laboratories and reported as positive or negative. HER2 gene amplification was determined by fluorescence in situ hybridisation (FISH) using the PathVysion kit (Vysis, Downers Grove, IL, USA) at Quest Diagnostics.

\section{Mutation assessments}

DNA was extracted from FFPE tumour tissue; seven of the exons comprising the kinase domain of PIK3CA (exons 14-20) and exon 9 (helical domain) were amplified by polymerase chain reaction and sequenced using the ABI3730XL sequencer (Applied Biosystems, Foster City, CA, USA); somatic single nucleotide polymorphisms (SNPs) were identified by sequence analysis and mapping the results to the PIK3CA GenBank reference sequence NM_006218.

\section{HER2 extracellular domain}

Serial blood samples were collected at baseline, every 4 weeks, and at study conclusion. Serum HER2 extracellular domain (ECD) levels were analysed at Quest Diagnostics using an HER-2/neu Microtiter enzyme-linked immunosorbent assay kit from Oncogene Science (Cambridge, MA, USA).

\section{Statistical design and analysis}

The hypotheses for both studies were based on expected ORR $(\geqslant 15 \%)$, accordingly the sample sizes (calculation using SAS version 8.2 , UNIX/Solaris) ensured $\geqslant 74 \%$ power to deem lapatinib monotherapy effective by correctly rejecting the null hypothesis (H0: $P<0.05)$ and accepting the alternative hypothesis (H1: $P>0.15)$. The efficacy endpoints were estimated using two-sided 95\% confidence interval (CI); TTP and OS were summarised using the Kaplan-Meier method.

Biomarkers were related to TTP using Cox proportional hazards models, and to clinical response using logistic regression. Correlations between biomarkers were evaluated using Spearman's rank correlation coefficient. Statistical tests were considered significant if $P<0.05$. Analyses were conducted using SAS v9.1.3.

\section{RESULTS}

\section{Patients}

Between June 2004 and October 2006, 122 patients (64 and 58 from EGF100642 and EGF104911, respectively) were enroled; 100 had 
Table I Patient characteristics - demographics and disease history

\begin{tabular}{|c|c|c|c|}
\hline Characteristic & $\begin{array}{c}\text { HER2 } \\
\text { positive } \\
n=100\end{array}$ & $\begin{array}{c}\text { HER2 } \\
\text { negative } \\
n=22\end{array}$ & $\begin{array}{c}\text { Total } \\
\text { population } \\
n=122\end{array}$ \\
\hline Median age, years & 55.0 & 52.5 & 55.0 \\
\hline \multicolumn{4}{|l|}{ ECOG PS, $n$ (\%) } \\
\hline $0-1$ & $97(97)$ & $19(86)$ & $116(95)$ \\
\hline 2 & $3(3)$ & $3(14)$ & $6(5)$ \\
\hline \multicolumn{4}{|l|}{ Disease stage, $n$ (\%) } \\
\hline$\|\mathrm{II} /\| \mathrm{II}(\mathrm{T} 4)$ & I ( 1$)$ & $2(9)$ & $3(2)$ \\
\hline IV & $99(99)$ & $20(91)$ & $119(98)$ \\
\hline \multicolumn{4}{|l|}{ Prior anti-tumour therapy, n (\%) } \\
\hline Anthracyclines & $100(100)$ & $22(100)$ & $122(100)$ \\
\hline Taxanes & $100(100)$ & $22(100)$ & $122(100)$ \\
\hline Trastuzumab & $100(100)$ & 0 & $100(82)$ \\
\hline Neoadjuvant ${ }^{\mathrm{a}}$ & $2(2)$ & 0 & $2(2)$ \\
\hline Adjuvant ${ }^{\mathrm{b}}$ & $4(4)$ & 0 & $4(3)$ \\
\hline Advanced/metastatic & $98(98)$ & 0 & $98(80)$ \\
\hline \multicolumn{4}{|l|}{ No. of metastatic sites, n (\%) } \\
\hline$\geqslant 3$ & $43(43)$ & $9(4 \mid)$ & $52(43)$ \\
\hline 2 & $36(36)$ & $6(27)$ & $42(34)$ \\
\hline 1 & $21(21)$ & $7(32)$ & $28(23)$ \\
\hline \multicolumn{4}{|l|}{ Hormone receptor status, n (\%) } \\
\hline ER+ and/or PgR+ & $38(38)$ & $14(64)$ & $52(43)$ \\
\hline$E R-$ and PgR- & $60(60)$ & $8(36)$ & $68(56)$ \\
\hline Unknown (ER and/or PgR) & $2(2)$ & 0 & $2(2)$ \\
\hline
\end{tabular}

Abbreviations: ECOG PS = European Cooperative Oncology Group Performance Status; $E R=$ oestrogen; $P g R=$ progesterone. ${ }^{\text {aP }}$ atients received trastuzumab for neoadjuvant and advanced/metastatic setting. ${ }^{\text {'T }}$ wo patients received trastuzumab for adjuvant and advanced/metastatic disease, two patients received in adjuvant setting only.

HER2-positive tumours (defined as IHC3 + or IHC2 + and FISH positive) and 22 (cohort B) had HER2-negative tumours (defined as IHC $0 / 1+$ or FISH negative). Although the planned patient number for cohort B was 60, accrual stopped based on minimal efficacy of lapatinib monotherapy reported in HER2-negative BC (Burstein et al, 2008). Out of 122 patients, 119 discontinued therapy; 108 (91\%) because of disease progression, 8 (7\%) because of AEs and $3(3 \%)$ withdrew consent. Three patients with HER2positive tumours remained on study treatment.

Patient baseline characteristics are shown in Table 1. Over $80 \%$ had at least three prior anti-tumour regimens. All patients received prior anthracyclines and taxanes. All but $2 \%$ of HER2-positive patients had previously received trastuzumab for advanced or metastatic disease. The majority $(75 \%)$ had received trastuzumab within 8 weeks before enrolment.

The median duration of treatment was 13.9 weeks in patients with HER2-positive tumours and 7.4 weeks in those with HER2negative tumours.

\section{Efficacy}

In HER2-positive patients, $1 \mathrm{CR}(1 \%)$ and 18 PRs (18.0\%) were documented, for an ORR of $19.0 \%$ (95\% CI: 11.8-28.1); SD and progressive disease (PD) were observed in $38 \%$ (38 out of 100) and $41 \%$ (41 out of 100), respectively; two patients had an unknown response. In patients with HER2-negative tumours only one patient responded $(\mathrm{CR} ; \mathrm{ORR}=4.5 \%)$, the majority $(68 \%)$ had $\mathrm{PD}$ and $14 \%$ had short-term SD. CBR in HER2-positive patients was $25.0 \%$ (95\% CI: $16.9-34.7$ ) and $4.5 \%$ in HER2-negative patients. The median TTP was 13.0 weeks $(25$ th, 75 th percentiles: $7.8,24.1)$ in HER2-positive patients and 8.0 weeks (25th, 75th percentiles:
5.4, 8.4) in HER2-negative patients. The median OS was 58.3 weeks (25th, 75th percentiles: 26.2, 64.8) in HER2-positive patients and 40.0 weeks $(25$ th, 75 th percentiles: $18.3,69.3$ ) in HER2-negative patients.

In the HER2-positive group, 10 patients had brain metastases at baseline, 8 had no brain lesion progression of these 2 had ORR of $\mathrm{PR}$, whereas brain lesions progressed in 2 patients.

\section{Safety}

Among 122 patients evaluable for safety, 120 (98.4\%) experienced at least $1 \mathrm{AE}$, most were Grade 1 or 2 . The Grade 4 AEs (increased bilirubin and $\gamma$-glutamyl transferase) occurred in a patient with concurrent progression of liver metastases. The AEs with $\geqslant 20 \%$ incidence are presented in Table 2.

There were 27 non-fatal serious AEs in 19 patients, 13 were possibly related to lapatinib. The only lapatinib-related serious AEs occurred in more than one patient were anorexia $(n=3,2.5 \%)$ and left ventricular dysfunction $(n=2,1.6 \%)$. Both patients experiencing left ventricular dysfunction remained on lapatinib and the event later resolved.

Three patients died because of disease progression. One patient experienced fatal AEs (increase in $\gamma$-glutamyl transferase and bilirubin); the events, deemed unrelated to lapatinib, were due to liver metastases progression. The other two experienced 'unexpectedly rapid' disease progression; one in a HER2-negative patient.

\section{Biomarkers}

The following biomarkers had results available in $95-100 \%$ of tumour samples: EGFR; HER2 (Table 3); ER; PgR. ErbB3, PTEN, IGF1R and BCL2 were well represented for EGF100642 $(>80 \%)$, but were sparse for EGF104911 ( $\leqslant 40 \%)$. The remaining biomarkers: Heregulin, TUNEL and Survivin, were represented in 55\% or fewer samples, with particular scarcity in EGF104911 ( <20\%).

The HER2 IHC score was strongly associated with TTP $(P=0.0083$, Figure 1$)$. TTP increased monotonically with increasing HER2 IHC score. CBR was higher in HER2 IHC $2+$ and $3+$ tumours $(P=0.0168)$. The proportion of patients deriving clinical benefit from lapatinib in IHC $0,1+, 2+$ and $3+$ groups was $4 \%$ (1 patient), $0 \%, 23 \%$, and $73 \%$, respectively. In $23 \%$ of patients experiencing clinical benefit from lapatinib and with IHC2 + tumours, all were HER2 FISH positive.

TTP and CBR differed between the studies. HER2-positive patients in EGF100642 experienced longer TTP compared with EGF104911 $(P=0.0212$, Figure 2). Cohort A (EGF100642) had a higher CBR than EGF104911 ( $P=0.0385$, Table 4$)$. The efficacy differences between the studies may be explained by the difference in the frequency of ER and PgR expression. In EGF100642, 17\% of HER2-positive tumours were ER positive, whereas $43 \%$ were ER positive in EGF104911; PgR positive was detected in 15\% and 35\%, respectively. Although CBR was not significantly associated with hormone receptor status $(P=0.100)$, PgR status had a significant effect on TTP $(P=0.016)$, whereas ER status did not $(P=0.3188)$. Patients with PgR-positive tumours regardless of ER status ( $n=26)$ had the shortest median TTP (8.4 weeks), whereas patients with PgR-negative tumours had similar median TTP (15.4 weeks for ERnegative, PgR negative, $n=60 ; 17$ weeks for ER positive, PgR negative, $n=12$ ).

EGFR and IGF1R expression were not significantly associated with efficacy parameters. IGF1R expression did not preclude patients from deriving benefit from lapatinib $(n=58, P=0.3394$, Figure 3A). PTEN expression did not associate with lapatinib response; in patients with HER2-positive tumours (EGF100642, $n=36)$, CBR was not dependent on PTEN $(P=0.9093)$, thus patients with tumours expressing low levels of PTEN derived benefit from lapatinib (Figure 3B). 
Table 2 Incidence of common adverse events reported in $\geqslant 20 \%$ of patients by maximum NCl CTCAE toxicity grade

\begin{tabular}{|c|c|c|c|c|}
\hline \multirow[b]{2}{*}{ MedDRA preferred term } & \multicolumn{2}{|c|}{ All grades } & \multicolumn{2}{|c|}{ Grades 3/4 } \\
\hline & No patients & $\%$ & Number of patients & $\%$ \\
\hline Diarrhoea & 86 & 70.5 & 7 & 5.7 \\
\hline Rash & 59 & 48.4 & I & 0.8 \\
\hline Stomatitis & 54 & 44.3 & 0 & 0 \\
\hline Nausea & 48 & 39.3 & । & 0.8 \\
\hline Pruritus & 36 & 29.5 & 0 & 0 \\
\hline Nasopharyngitis & 33 & 27.0 & 0 & 0 \\
\hline Aspartate aminotransferase increased & 28 & 23.0 & 5 & 4.1 \\
\hline Dry skin & 27 & 22.1 & 0 & 0 \\
\hline Vomiting & 27 & 22.1 & 2 & 1.6 \\
\hline Pyrexia & 24 & 19.7 & I & 0.8 \\
\hline Weight decreased & 22 & 18.0 & 0 & 0 \\
\hline
\end{tabular}

Abbreviations: MedDRA = Medical Dictionary for Regulatory Activities; NCI CTCAE = National Cancer Institute Common Terminology for Adverse Events.

Table 3 Distribution of EGFR and HER2 biomarker IHC scores

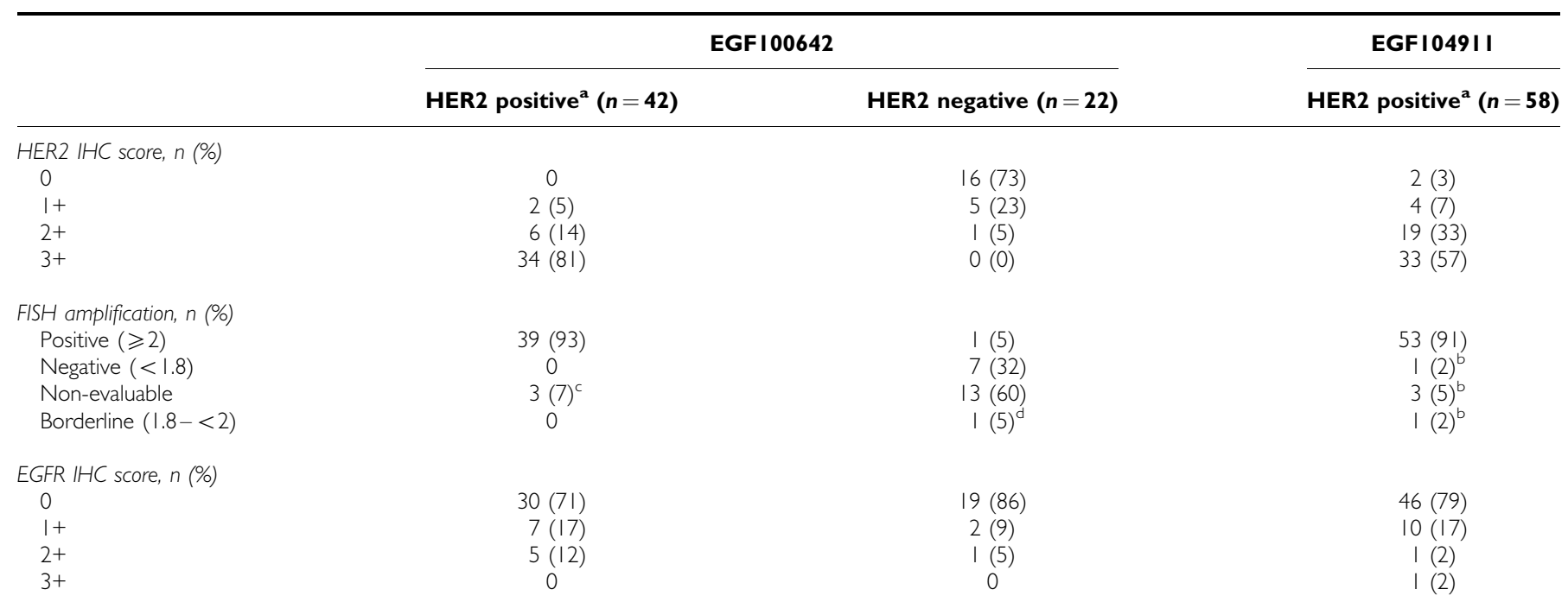

a HER2 eligibility was determined by site local laboratory data. ${ }^{b} \mathrm{FISH}$-negative tumour was $\mathrm{HC} 0$; non-evaluable: one was $\mathrm{HC} 0$, one was IHCI+, one was IHC3+; borderline was $\mathrm{HHC2}+$. ${ }^{\mathrm{F}} \mathrm{FSH}$ non-evaluable tumours: one was $\mathrm{HHCl}+$, one was $\mathrm{HC} 2+$, one was $\mathrm{HC} 3+$. ${ }^{\mathrm{F}} \mathrm{FISH}$ borderline tumour was $\mathrm{IHC} 0$.

For exploratory purposes, biomarkers were tested for correlation with each other in the HER2-positive patients. Significant correlations were found between BCL2 and IGF1R $(r=0.33$, $P=0.015)$, ErbB3 and Heregulin $(r=0.39, P=0.012)$ and IGF1R and PTEN $(r=0.62, P=0.0003)$.

Biomarkers were compared with ER and PgR status in HER2positive patients using the Wilcoxon rank sum test. BCL2 levels were significantly higher in ER-positive patients $(n=52, P=0.017)$ and PgR-positive patients $(n=51, P=0.025)$. Heregulin $(n=40$, $P=0.005)$ and Survivin $(n=22, P=0.047)$ were found to be higher in PgR-negative patients.

PIK3CA gene (exon 9; 14-20) mutation analysis was conducted in EGF100642; 30 tumour samples had sufficient quantities for of genomic DNA. Owing to the low quantities of genomic DNA in all tumour samples, exon 20 was the highest priority because of the presence of the well-characterised H1047R kinase-activating mutation located in this exon and detected in BC (Kang et al, 2005). Exon 20 mutation analysis was completed in 29 samples; in 3 tumours the H1047R mutation was detected. Lapatinib response in the three patients (HER2 positive) whose tumours harboured the H1047R mutation included one durable PR ( 32 weeks), and two SD (16 weeks). Mutation analysis on the remaining exons identified two SNPs in two different tumour samples resulting in nonconservative amino acid substitutions (exon 15: E767K; exon 18: T898I). Although both substitutions were predicted to be structurally tolerated and speculated to not directly affect PIK3CA kinase activity, the indirect functional implications were unknown. The patients whose tumours contained these SNPs derived clinical benefit from lapatinib: one (HER2 positive) SD for $\geqslant 24$ weeks; one (HER2 negative) CR for 23 weeks. In tumours with evaluable results (10 out of 30 ), no SNPs resulting in an amino acid change were detected in exon 9.

The distribution of baseline serum ECD (bECD) values showed that levels appear to be higher in patients with HER2-positive tumours (median: $44.5 \mathrm{ng} \mathrm{ml}^{-1}$; 25th, 75th percentiles: $15.2,114.3$ ) vs HER2-negative tumours (median: $9.9 \mathrm{ng} \mathrm{ml}^{-1}$; 25 th, 75 th percentiles: $8.0,12.4$ ); however, bECD did not predict response. After adjusting for HER2 status in the pooled sample, bECD (entered 


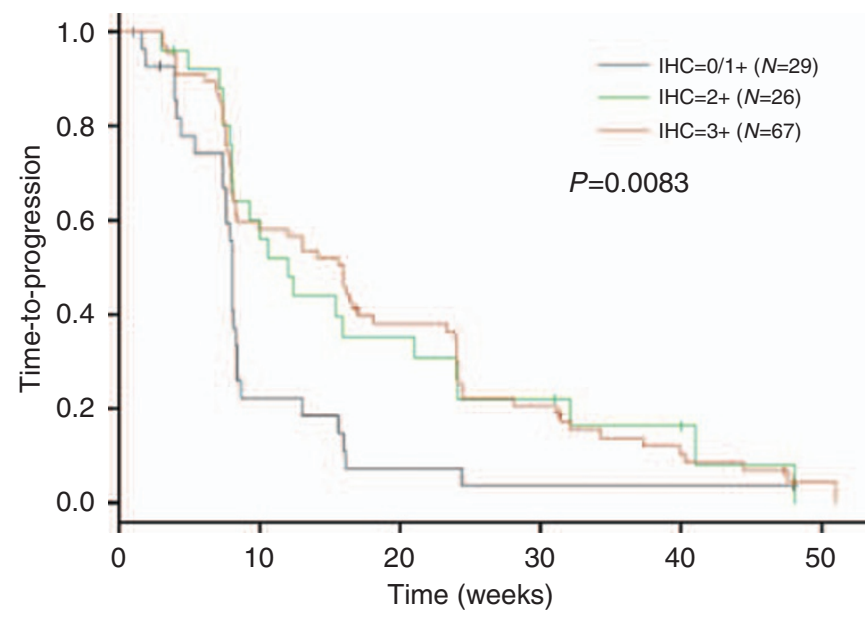

Figure I Kaplan-Meier estimates for TTP in the combined studies. TTP is stratified by HER2 IHC Score. Median IHC 0/I = 8 weeks; IHC $2+=12$ weeks; $I H C 3+=16$ weeks. (log rank $P=0.0083$ ); 23 out of 26 HER2 $\mathrm{IHC2}+$ tumours were FISH positive, 3 out of 26 tumours were nonevaluable by FISH.

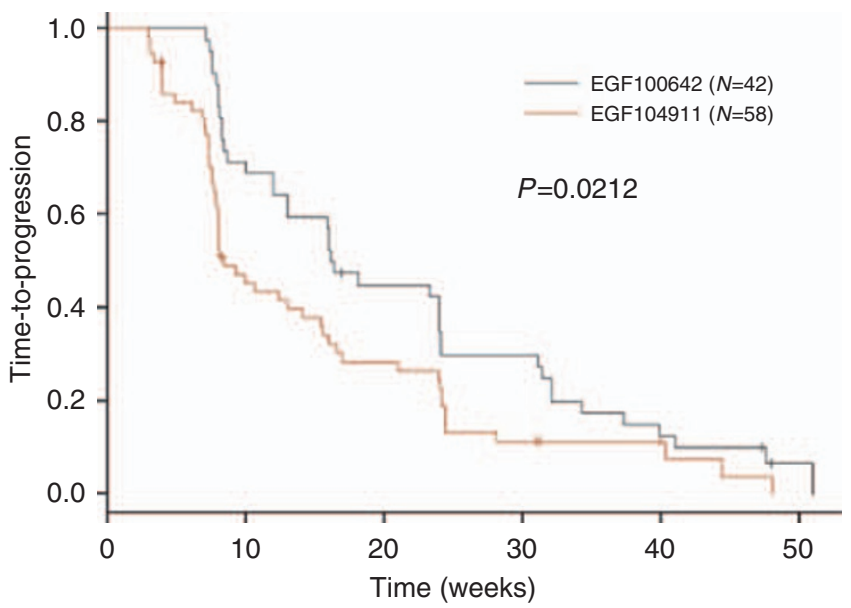

Figure 2 Kaplan-Meier estimates for TTP. TTP is significantly longer in EGFI00642 HER2-positive patients vs EGFI049 I I patients (median TTP for EGFI00642 = | 6.3 weeks; EGFI049| | = 8.4 weeks; $P=0.0212$ ).

into the model as log of bECD) was not a significant predictor of TTP in a proportional hazards model $(P=0.568)$. In a similar model, neither were bECD levels predictors of TTP within HER2negative $(P=0.531)$ or HER2-positive patients $(P=0.582)$, nor were bECD levels predictors of TTP in the HER2-positive patients when adjusted for ER/PgR status $(P=0.896)$.

\section{DISCUSSION}

Lapatinib monotherapy was efficacious in treating HER2-overexpressing advanced or $\mathrm{MBC}$ that relapsed after trastuzumab treatment. Lapatinib benefit was confined mostly to HER2-positive tumours (ORR was $19 \%$; CBR was $25.0 \%$ ) and confirmed previous reports (Geyer et al, 2006; Gomez et al, 2008). In this heavily pretreated population with refractory HER2-positive BC, treatment with single-agent lapatinib resulted in a median TTP of 13.0 weeks and median OS of 58.3 weeks. Median progression-free survival was identical to TTP (13.0 weeks for the HER2-positive
Table 4 Best response by study and cohort

\begin{tabular}{|c|c|c|c|}
\hline & \multicolumn{2}{|c|}{ EGF 100642} & \multirow{2}{*}{$\begin{array}{c}\text { EGF } 1049 \text { I I } \\
\begin{array}{l}\text { HER2 positive } \\
(n=58)\end{array}\end{array}$} \\
\hline & $\begin{array}{l}\text { HER2 positive } \\
(n=42)\end{array}$ & $\begin{array}{c}\text { HER2 negative } \\
(n=22)\end{array}$ & \\
\hline \multicolumn{4}{|c|}{ Best response, $n$ (\%) } \\
\hline$C R$ & 0 & । (5) & I (2) \\
\hline PR & $10(24)$ & 0 & $8(14)$ \\
\hline SD & $20(48)$ & $3(14)$ & $18(31)$ \\
\hline PD & $12(29)$ & $15(68)$ & $29(50)$ \\
\hline $\mathrm{NE}$ & 0 & $3(14)$ & $2(3)$ \\
\hline CBR & $16(38)$ & ( (5) & $11(19)$ \\
\hline
\end{tabular}

Abbreviations: $C R=$ complete response; $P R=$ partial response; $\mathrm{SD}=$ stable disease; $\mathrm{PD}=$ progressive disease; $\mathrm{NE}=$ non-evaluable; $\mathrm{CBR}=$ clinical benefit rate $(\mathrm{CR} ; \mathrm{PR}$; $\mathrm{SD} \geqslant 24$ weeks).

population), as no time-to-event at the time of data cut-off occurred because of death.

TTP and CBR differed between the studies. Patient characteristics were similar in both studies except for differences observed in the distribution of patients with hormone-receptor-positive BC, although expression was not confirmed by central review. This small dataset suggests that lapatinib may affect TTP based on PgR status. PgR expression and activity, whereas dependence on classical ER genomic signalling has been shown to be regulated by growth factor signalling (Arpino et al, 2008). Additional reports have suggested that negative PgR expression indicates high growth factor activity (Cui et al, 2005). Perhaps in the context of HER2 overexpression and ER positivity, low or absent PgR expression may be a predictor of tumour dependence on HER2 activity and thus sensitivity to HER2 inhibition with agents such as lapatinib.

Lapatinib response may be independent of PTEN, PIK3CA and IGF1R, as supported by lapatinib responses observed in these studies and demonstrated in preclinical studies (Nahta et al, 2007; Xia et al, 2007). Patients with HER2-positive tumours having PIK3CA amino acid changes derived benefit from lapatinib. Additionally, patients with low PTEN, or expressing IGF1R also derived benefit from lapatinib. These findings, albeit in a small cohort, suggested that lapatinib anti-tumour activity may depend on a cellular milieu different from trastuzumab, which reportedly has decreased activity in tumours with the aforementioned characteristics (Nahta and Esteva, 2006). Ongoing clinical trials in early and advanced $\mathrm{BC}$ have been designed to elucidate lapatinib mechanism of action, identify predictive biomarkers and further define the differences in activity compared with other HER2 inhibitors.

In the HER2-negative cohort, one patient experienced a durable CR ( 23 weeks) and had a primary tumour with the following molecular characteristics: HER2 negative and EGFR negative; ER positive and PgR positive; low expression of PTEN and PIK3CA (E767K) SNP. Biomarker evaluations were performed on the primary tumour and not the recurrent site; hence, it is interesting to speculate that the genotype/phenotype of treated tumour was modified at recurrence, supporting the practicing biopsy on relapse.

Brain metastases is an area of significant unmet medical need, as up to one-third of HER2-overexpressing MBC patients develop central nervous system metastases after trastuzumab treatment (Bendell et al, 2003; Clayton et al, 2004). In this study, 10 patients had brain metastases at baseline and of these 8 experienced either systemic PR or SD, indicating that lapatinib monotherapy is efficacious in HER2-positive BC patients with brain metastases, thus supporting previous reports (Lin et al, 2008).

Trastuzumab treatment is associated with cardiac dysfunction, with up to $28 \%$ of patients experiencing such events (Guarneri et al, 2006), although asymptomatic LVEF declines were more 
A

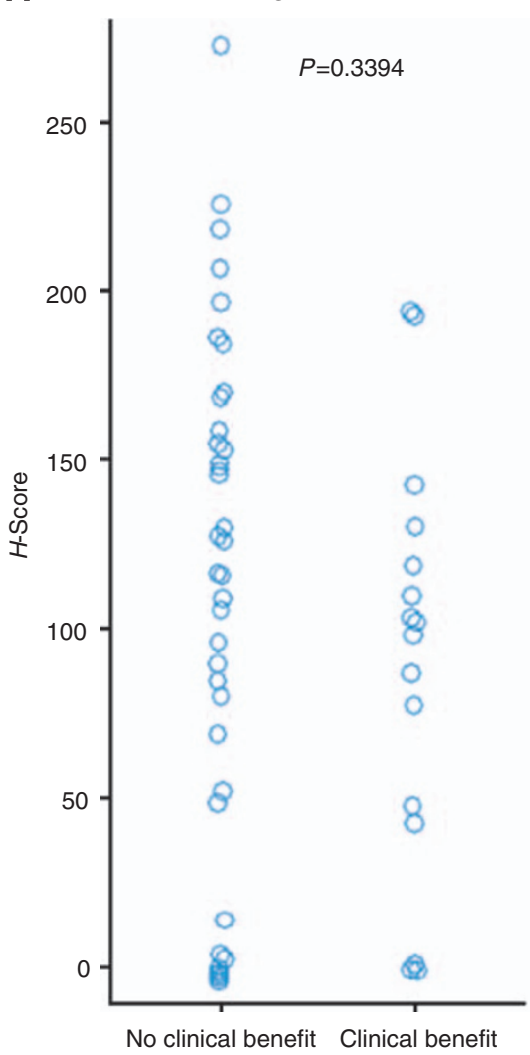

B

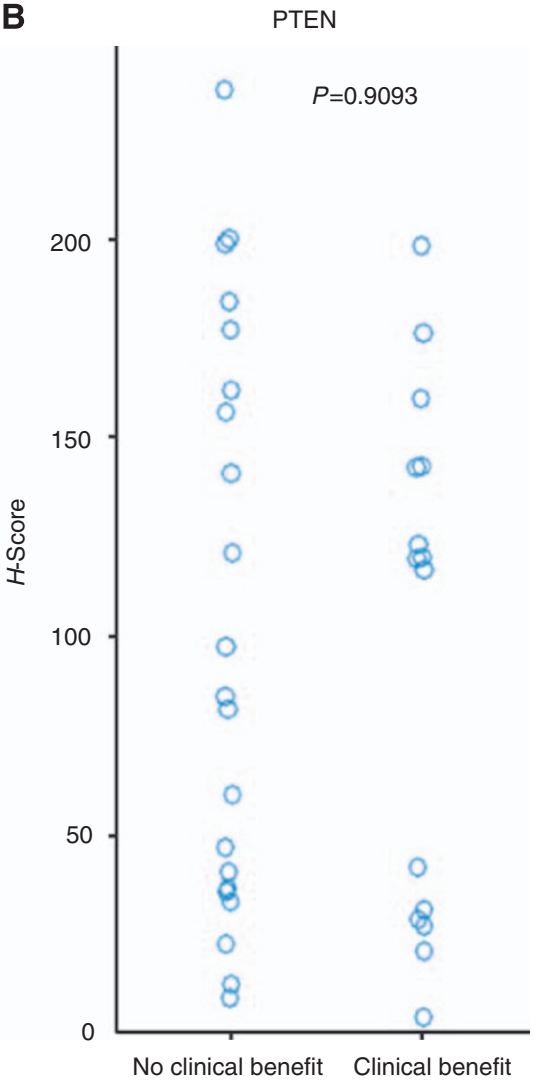

Figure 3 Distribution of $\mathrm{H}$-scores as measured by $I H C$, grouped according to patient response (clinical benefit $=\mathrm{CR}$; $P R$; s.d. $\geqslant 24$ weeks; no clinical benefit =s.d. $<24$ weeks; PD; NE). (A) IGFIR protein expression levels $(n=58 ; P=0.3394)$. (B) PTEN protein expression levels $(n=36 ; P=0.9093)$.

common than symptomatic heart failure (Marty et al, 2003). In this study, three patients experienced LVEF decrease (2.4\%); however, with mild severity (Grade 1). These data were similar to that observed from 44 lapatinib clinical trials, in which the incidence was reported as $1.6 \%$ (60 out of 3689 lapatinib-treated patients) (Perez et al, 2008).

The results in Japanese patients showed that lapatinib was well tolerated and an effective treatment for HER2-positive BC $(\mathrm{ORR}=19 \%$; $\mathrm{CBR}=25 \%)$, despite disease progression on trastuzumab-based therapy. Although low ORR (4.3; 7.7\%) were observed in similar patient populations who were mostly Caucasian (Burstein et al, 2008; Blackwell et al, 2009); however, neither intrinsic nor extrinsic factors were evaluated to determine the efficacy differences. In first-line patients with trastuzumabnaïve, HER2-positive advanced or MBC, lapatinib monotherapy resulted in 24\% ORR (Gomez et al, 2008). Lapatinib combination therapy has promising efficacy as treatment for HER2-positive advanced or MBC, including treatment-naïve MBC (Geyer et al, 2006; Di Leo et al, 2008; O’Shaughnessy et al, 2008; Johnston et al, $2008 \mathrm{a}, \mathrm{b})$. The outcome of this study complements the efficacy reported earlier for lapatinib and confirms that lapatinib offers a beneficial therapeutic option to patients with HER2-overexpressing
$\mathrm{BC}$, including those with brain metastases. The efficacy for the tumours having the altered PI3K pathway may provide a novel possibility in the treatment of HER2-positive BCs.

\section{ACKNOWLEDGEMENTS}

We thank the women who participated in these studies and their families; the investigators, in particular, Hirofumi Fujii, Yasuhiro Yamanaka, Hiroshi Sonoo, Muneaki Sano, Nobuaki Sato, Norikazu Masuda, Shinji Ohno, Keisei Anan, Hiroko Bando, Tsunehiro Nishi, Hironobu Minami, Tadashi Kobayashi, Taro Asaga, Kazuo Tamura and Masaru Narabayashi; medical, nursing and research staff at the study centres. We also thank Giuseppe Viale, Ganesh Sathe, Anne-Marie Martin, Louise Downie, Kevin Jackson and Tona Gilmer for their technical expertise with the biomarker studies. This work was sponsored by GlaxoSmithKline K.K.

\section{Conflict of interest}

The authors declare no conflict of interest.

\section{REFERENCES}

Arpino G, Wiechmann C, Osborne CK, Schiff R (2008) Crosstalk between the estrogen receptor and the HER tyrosine kinase receptor family: molecular mechanism and clinical implications for endocrine therapy resistance. Endocr Rev 29: 217-233

Bendell J, Domchek S, Burstein H, Harris L, Younger J, Kuter I, Bunnell C, Rue M, Gelman R, Winer E (2003) Central nervous system metastases in women who receive trastuzumab-based therapy for metastatic breast carcinoma. Cancer 97: 2972-2977

Blackwell K, Pegram M, Tan-Chiu E (2009) Single-Agent lapatinib for the treatment of HER2-overexpressing advanced or metastatic breast cancer that progressed on first- or second-line trastuzumab containing regimens. Ann Oncol 20: 1026-1031 
Burstein HJ, Storniolo AM, Franco S, Forster J, Stein S, Rubin S, Salazar VM, Blackwell KL (2008) A phase II study of lapatinib monotherapy in chemotherapy-refractory HER2-positive and HER2-negative advanced or metastatic breast cancer. Ann Oncol 19: 1068 - 1074

Clayton AJ, Danson S, Jolly S, Ryder WD, Burt PA, Stewart AL, Wilkinson PM, Welch RS, Magee B, Wilson G, Howell A, Wardley AM (2004) Incidence of cerebral metastases in patients treated with trastuzumab for metastatic breast cancer. Br J Cancer 91: 639-643

Cobleigh MA, Vogel CL, Tripathy D, Robert NJ, Scholl S, Fehrenbacher L, Wolter JM, Paton V, Shak S, Lieberman G, Slamon DJ (1999) Multinational study of the efficacy and safety of humanized anti-HER2 monoclonal antibody in women who have HER2-overexpressing metastatic breast cancer that has progressed after chemotherapy for metastatic disease. J Clin Oncol 17: 2639-2648

Cui X, Schiff R, Arpino G, Osborne CK, Lee AV (2005) Biology of progesterone receptor loss in breast cancer and its implications for endocrine therapy. J Clin Oncol 23: $7721-7735$

Di Leo A, Gomez HL, Aziz Z, Zvirbule Z, Bines J, Arbushites MC, Guerrera SF, Koehler M, Oliva C, Stein SH, Williams LS, Dering J, Finn RS, Press MF (2008) Phase III, double-blind, randomized study comparing lapatinib plus paclitaxel with placebo plus paclitaxel as first-line treatment for metastatic breast cancer. J Clin Oncol 26: 5444-5552

Gomez H, Doval D, Chavez M, Ang PC, Aziz Z, Nag S, Ng C, Franco SX, Chow LW, Arbushites MC, Casey MA, Berger MS, Stein SH, Sledge GW (2008) Efficacy and safety of lapatinib as first-line therapy for HER2amplified locally advanced or metastatic breast cancer. J Clin Oncol 26: 2999- 3005

Guarneri V, Lenihan DJ, Valero V, Durand JB, Broglio K, Hess KR, Michaud LB, Gonzalez-Angulo AM, Hortobagyi GN, Esteva FJ (2006) Long-term cardiac tolerability of trastuzumab in metastatic breast cancer: the M.D. Anderson Cancer Center experience. J Clin Oncol 24: 4107-4115

Geyer CE, Forster J, Lindquist D, Chan S, Romieu G, Peinkowski T, JagielloGruszfeld A, Crown J, Chan A, Kaufman B, Skarlos D, Campone M, Davidson N, Berger M, Oliva C, Rubin SD, Stein S, Cameron D (2006) Lapatinib plus capecitabine for HER2-positive advanced breast cancer. N Engl J Med 355: 2733 - 2744

Johnston S, Trudeau M, Kaufman B, Boussen H, Blackwell K, LoRusso P, Lombardi DP, Ben Ahmed S, Citrin DL, DeSilvio ML, Harris J, Westlund RE, Salazar V, Zaks TZ, Spector NL (2008a) Phase II study of predictive biomarker profiles for response targeting human epidermal growth factor receptor 2 (HER-2) in advanced inflammatory breast cancer with lapatinib monotherapy. J Clin Oncol 26: 1066-1072

Johnston S, Pegram M, Press M, Pippen J, Pivot X, Gomez H, Florance A, O'Rourke L, Maltzman J (2008b) Lapatinib combined with letrozole vs letrozole alone for front line postmenopausal hormone receptor positive $(\mathrm{HR}+)$ metastatic breast cancer $(\mathrm{MBC})$ : first results from the EGF30008 Trial. 31st Annual San Antonio Breast Cancer Symposium (SABCS): Abstract 46

Kang S, Bader A, Vogt P (2005) Phosphatidylinositol 3-kinase mutations identified in human cancer are oncogenic. Proc Natl Acad Sci USA 102: $802-807$

Lin NU, Carey LA, Liu MC, Younger J, Come SE, Ewend M, Harris GJ, Bullitt E, Van den Abbeele AD, Henson JW, Li X, Gelman R, Burstein HJ Kasparian E, Kirsch DG, Crawford A, Hochberg F, Winer EP (2008)
Phase II trial of lapatinib for brain metastases in patients with human epidermal growth factor receptor 2-positive breast cancer. J Clin Oncol 26: 1993 - 1999

Marty M, Baselga J, Gatzemeier U (2003) Pooled analysis of six trials of trastuzumab (Herceptin): exploratory analysis of changes in left ventricular ejection fraction (LVEF) as a surrogate for clinical cardiac events (abstract). Breast Cancer Res Treat 82: S47

McCarty Jr KS, Szabo E, Flowers JL, Cox EB, Leight GS, Miller L, Konrath J, Soper JT, Budwit DA, Creasman WT, Seigler HF, McCarty Sr KS (1986) Use of a monoclonal anti-estrogen receptor antibody in the immunohistochemical evaluation of human tumors. Cancer Res 46(suppl 8): 4244s-44248s

Montemurro F, Donadio M, Clavarezza M, Redana S, Jacomuzzi ME, Valabrega G, Danese S, Vietti-Ramus G, Durando A, Venturini M, Aglietta M (2006) Outcome of patients with HER2-positive advanced breast cancer progressing during trastuzumab-based therapy. Oncologist 11: $318-324$

Mukherjee A, Dhadda AS, Shehata M, Chan S (2007) Lapatinib: a tyrosine kinase inhibitor with a clinical role in breast cancer. Expert Opin Pharmacother 8: 2189-2204

Nahta R, Esteva F (2006) Molecular mechanisms of trastuzumab resistance. Breast Cancer Res 8: 215

Nahta R, Yuan LX, Du Y, Esteva FJ (2007) Lapatinib induces apoptosis in trastuzumab-resistant breast cancer cells: effects on insulin-like growth factor 1 signaling. Mol Cancer Ther 6: 667-674

O'Shaughnessy J, Blackwell KL, Burstein H, Storniolo AM, Sledge G, Baselga J, Koehler M, Laabs S, Florance A, Roychowdhury D (2008) A randomized study of lapatinib alone or in combination with trastuzumab in heavily pretreated HER2+ metastatic breast cancer progressing on trastuzumab therapy. J Clin Oncol 26 abstr 1015

Perez EA, Koehler M, Byrne J, Preston AJ, Rappold E, Ewer MS (2008) Cardiac safety of lapatinib: pooled analysis of 3689 patients enrolled in clinical trials. Mayo Clin Proc 83: 679-686

Slamon DJ, Clark GM, Wong SG, Levin WJ, Ullrich A, McGuire WL (1987) Human breast cancer: correlation of relapse and survival with amplification of the HER-2/neu oncogene. Science 235: 177-182

Suter TM, Procter M, van Veldhuisen DJ, Muscholl M, Bergh J, Carlomagno C, Perren T, Passalacqua R, Bighin C, Klijn JG, Ageev FT, Hitre E, Groetz J, Iwata H, Knap M, Gnant M, Muehlbauer S, Spence A, Gelber RD, Piccart-Gebhart MJ (2007) Trastuzumab-associated cardiac adverse effects in the herceptin adjuvant trial. J Clin Oncol 25: 3859-3865

Vogel CL, Cobleigh MA, Tripathy D, Gutheil JC, Harris LN, Fehrenbacher L, Slamon DJ, Murphy M, Novotny WF, Burchmore M, Shak S, Stewart SJ, Press M (2002) Efficacy and safety of trastuzumab as a single agent in first-line treatment of HER2-overexpressing metastatic breast cancer J Clin Oncol 20: 719-726

Xia W, Husain I, Liu L, Bacus S, Saini S, Spohn J, Pry K, Westlund R, Stein SH, Spector NL (2007) Lapatinib antitumour activity is not dependent upon phosphatase and tensin homologue deleted on chromosome 10 in HER2-overexpressing breast cancers. Cancer Res 67: 1170-1175

Xia W, Mullin RJ, Keith BR, Liu LH, Ma H, Rusnak DW, Owens G, Alligood KJ, Spector NL (2002) Anti-tumour activity of GW572016: a dual tyrosine kinase inhibitor blocks EGF activation of EGFR/HER2 and downstream Erk1/2 and AKT pathways. Oncogene 21: 6255-6263 